\title{
A Low-Complexity UEP Image Transmission Scheme over Satellite
}

\author{
Tao Ma, Zhanqi Xu, Xiaoyong Wei, Fan Yang, and Ding Zhe
}

\begin{abstract}
Image transmission over satellite is an important application in our life. The unequal error protection (UEP) fountain coding is able to avoid the backchannel retransmission, and also provides UEP for the embedded compressed multimedia data. Thus it has become the ideal solution for image transmission scheme over satellite. However, the complexity of the existing UEP fountain coding hinders the applications on energy-constraint satellite. In this paper, a low-complexity UEP image transmission scheme is proposed. Two elements are designed to reduce the complexity of our transmission scheme. First, a very low-complexity image coding algorithm, the improved SPIHT (ISPIHT), is adopted as our compression codec, which provides almost same compression performance as JPEG2000 but much fast coding speed. Second, the descent method based optimization algorithm is proposed to further simplify the computational complexity. With these two elements, our proposed UEP Image transmission scheme is very applicable for satellite communication. Finally, our simulation results show our low-complexity UEP image transmission scheme outperforms the traditional EEP scheme.
\end{abstract}

Index Terms-UEP, low-complexity, image transmission, fountain code.

\section{INTRODUCTION}

Multimedia transmission in the network has found many applications such as video broadcasting, video conferencing and video game, etc., and become more and more popular in our life. Multimedia transmission over satellite is able to provide the access to all kinds of multimedia applications for the area such as the ocean area or remote mountain area, where terrestrial network is unable to cover. Thus it becomes one of the important research topics.

In satellite network, back channel typically has high latency and limited capacity. However, multimedia communication usually requires real-time transmission and high bandwidth. Therefore, the typical feedback technique, which requires frequent retransmissions and high back channel capacity, is unsuitable for the satellite communication.

To eliminate the need for retransmission and to allow receivers to access data asynchronously, a digital fountain code technique is proposed [1]. A digital fountain injects a stream of distinct encoding packets into the network. Any receiver is able to reconstruct the source data from any subset

Manuscript received September 20, 2013; revised November 10, 2013. This work was supported in part by State High-Tech Development Plan "863 program" (2012AA01A505) and in part by the Advanced Research Program of Science and Technology of China (41001080201).

Tao Ma, Zhanqi Xu, Fan Yang and Zhe Ding are with the School of Telecommunications Engineering at Xidian University, Xian, China (e-mail: tma@xidian.edu.cn, zqxu@xidian.edu.cn).

Yongwei Xiao is with the 54th institute of Electronic and Technology Group of China, Shi Jia Zhuang, China. of the encoding packets equal in total length to the source data [2], [3]. This character makes fountain code is very suitable for the communication over satellite.

To further improve the performance of multimedia transmission over satellite, the traditional fountain code is modified to be able to provide unequal error protection (UEP) property in order to fit the characteristic of the compressed multimedia data.

Many fountain codes with UEP property have been proposed lately. It can be categorized into two classes: the weighted approach [4], [5] and the windowing approach [6]. In the weighted approach, the message block to be transmitted is partitioned into classes of different importance, and the different selection probabilities are assigned into these different classes in such a way that more important classes are more likely to be chosen in forming the output symbols, resulting in the UEP property. The basic idea of windowing approach is to encoding source data only in the selected window. The windows are predesigned in an embedded manner that the smaller windows are contained in the larger windows. The problem with the windowing approach is that the overall degree distribution is altered. It is well known that both performance and complexity are sensitive to the choice of the degree distributions. Therefore, without re-optimizing the degree distribution, the windowing approach may worsen the code behavior.

All above research work only provide the UEP framework for the general multimedia transmission. Furthermore, their optimization problem is very computational complex, and not applicable to energy constrained satellite communication.

In this paper, we address the complexity of the existing work. An applicable UEP image transmission scheme over satellite is proposed. First, the improved SPIHT (ISPIHT) [7], one of state of the art image codec, is adopted in our proposed scheme. The ISPIHT codec is the improved version of SPIHT codec, which has almost the same coding performance with that of the JPEG2000 but much lower computational complexity and memory requirements [8], [9]. Second, the compressed image is divided into two parts, which are selected with the different probabilities in LT code. Thus the complexity of the optimization problem in our scheme is simplified to be restrained only in two variables: the selection probability of the first part compressed image $\mathrm{p}_{1}$ and the size of the first part compressed image L. Furthermore, a descent method is adopted to find the optimal solution $\mathrm{p}_{1}$ in convex problem, resulting in low complexity of our UEP scheme. Finally the simulation results verify our proposed scheme outperforms the EEP scheme with a very low computational complexity. Thus it is very suitable for the image transmission over satellite.

The rest of paper can be outlined as follows. Section II 
gives the two background elements of our transmission: The embedded characteristic of ISIHPT codec and the theory of LT code are presented. Section III presents our low-complexity UEP image transmission scheme in detail. The simulation is conducted to verify our proposed scheme in Section IV. Finally the conclusion remark is given in Section V.

\section{BACKGROUND}

\section{A. The Dependency Relationship of the ISIHPT Coded Image}

In this section, we introduce dependency relationship of the ISPIHT codec.

In ISPIHT, an image is first wavelet transformed. The resulting wavelet coefficients are processed through set partition sorting and refining stages repeatedly with its threshold decreased by half at each bit-plane until the coding budget is fulfilled. In $i$ th bit-plane, the output of set partition sorting records the position information of wavelet coefficients in $i$ th bit-plane, and are denoted as $\mathrm{P}_{i}$. The output of the refining stages records value information of wavelet coefficients in all previous bit-planes, and are denoted as $\mathrm{V}_{1}, \cdots \mathrm{V}_{i}$. The dependency relationship of the wavelet confidents are illustrated as Fig. 1.

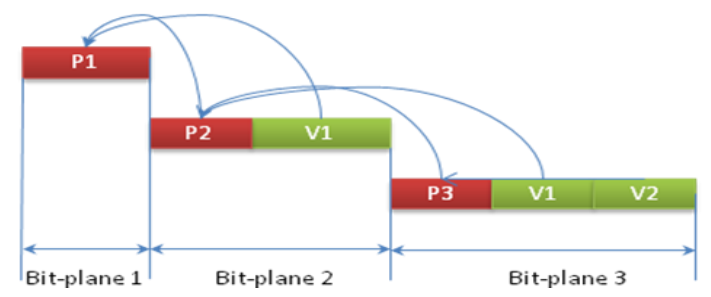

Fig. 1. Dependency relationship of the ISPIHT encoded image.

As Fig. 1 shows, the success of decoding wavelet coefficients in bit-plane $i+1$ is dependent on the success of decoding all the wavelet coefficients in previous $i$ bit-planes. That characteristic leads to the decease of the importance of encoded wavelet coefficients as the bit-plane number increase.

\section{B. LT Code with Unequal Error Protection Property}

In this section, we introduce the analytical basis of the LT code with unequal error protection, which is the theoretical foundation of our proposed UEP image transmission scheme [5].

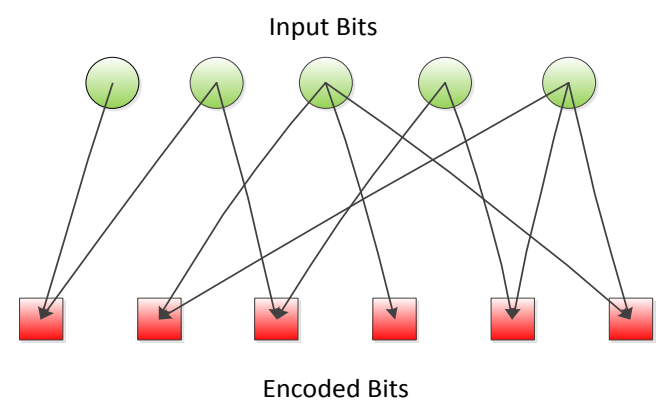

Fig. 2. LT coding process.

Suppose we have k bits message needed to be transmitted. In original LT coding process, which is illustrated in Fig. 2, a degree variable $\mathrm{d}$ is firstly generated according to one particular probability distribution. Second, d bits are uniformly selected from $k$ bits of the input message to generate a encode bit. This process is repeated until $(1+\varepsilon) k$ encoded bits are generated. $1+\varepsilon$ is the coding overhead. As we can see, the probability of selecting input bits to encode is uniformly distributed in the original LT coding. Therefore, it is an equal error protection (EEP) coding.

In more general case, if we select the input bits non-uniformly at random as Fig. 3 shows, the original LT code is extended from EEP to UEP.

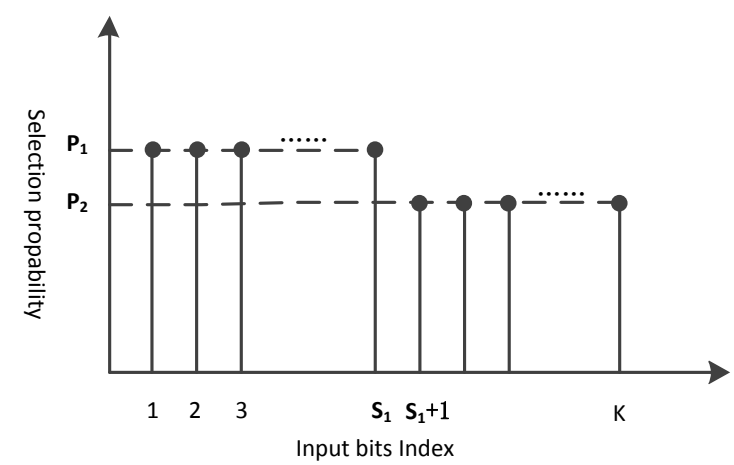

Fig. 3. LT coding with different selection probability.

We divided $\mathrm{k}$ bits input message into $\mathrm{r}$ sections. The input bits in different sections have different selection probabilities.

According to paper [4], if $r=2$, the probability that the bits in the first section is not recovered after i decoding iteration is

$$
y_{i, 1}=e^{\left.-k p_{1} \mu m \beta(1-(1-\alpha)) k p_{2} y_{i-1,1}-\alpha k p_{1} y_{i-1,1}\right)}
$$

And the probability that the bits in the second section is not recovered after $i$ decoding iteration is

$$
y_{i, 2}=e^{\left.-k p_{2} \mu \eta \beta(1-(1-\alpha)) k p_{1} y_{i-1,2}-\alpha k p_{1} y_{i-1,1}\right)}
$$

where $\beta(x)=\Omega^{\prime}(x) / \Omega^{\prime}(1), \mu=\Omega^{\prime}(1)$, and $y_{0,1}=y_{0,2}=0$. The number of input bit in the first section is $\alpha k$.

\section{OUR PROPOSED UEP IMAGE TRANSMISSION SCHEME}

In our UEP image transmission scheme, the image is first ISPIHT compressed. Then the compressed data is divided into two parts, which is shown in Fig. 4.

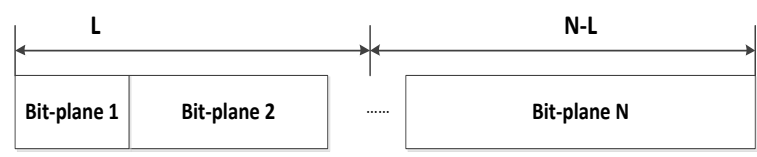

Fig. 4. Compressed Image bit stream.

The total number of bit-planes of compressed image is assumed to be N. We select the first L bit-planes as the first part. And the rest N-L are the second part. A UEP LT coding is applied to the compressed image bit-stream. The first $\mathrm{L}$ bit-planes 'bits are selected with probability of $p_{1}$, the rest bits are selected with probability of $p_{2}$. After LT encoding with UEP property, the encoded bit-stream are packetized and transmitted from satellite. The scheme is shown as Fig. 5. 


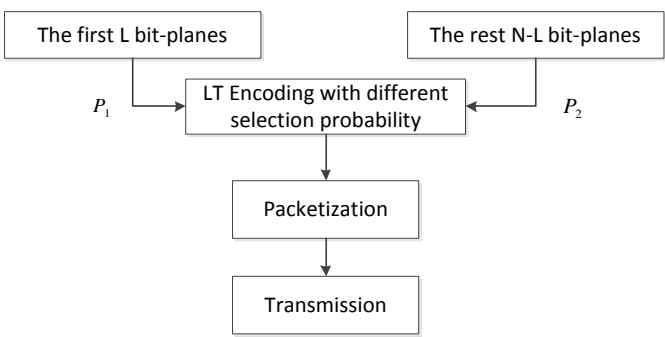

Fig. 5. The proposed UEP transmission scheme.

Let $k$ be the total number of compressed image bits. According to the dependence relationship of the bit-planes of image compressed data. The average image quality after decoding is the sum of all the possible image qualities weighted with their corresponding transmission success rate. Therefore, we express the average peek signal to noise ratio (PSNR) as the following equation,

$$
P S N R_{\text {avg }}=\sum_{i=1}^{L_{k}} P(i) P S N R(i)+\sum_{i=L_{k}+1}^{L_{N}} P(i) P S N R(i)
$$

where PSNR $(i)$ is the Peek Signal to Noise Ratio of the decoded image when first $i$ packets are received. $P(i)$ is probability of first $i$ packets are successfully received. $L k$ is the number of packets in the first part. LN is the total number of packets of the entire message.

According to our scheme, the encoded bit-stream is divided into two parts. The first part consists of the first $L k$ bits with a packet error rate (PER) of PER1, and the second part consists of the rest bits with a PER of PER2.Thus, $P(i)$ can be calculated as Equation 4 .

$$
p(i)= \begin{cases}\left(1-P E R_{1}\right)^{i} P E R_{1} & \text { if } i<L_{k} \\ \left(1-P E R_{1}\right)^{i}\left(1-P E R_{2}\right)^{i-L_{k}} P E R_{2} & \text { if } i>L_{k}\end{cases}
$$

where $\mathrm{PER}_{1}, \mathrm{PER}_{2}$ can be calculated as the following equations.

$$
\begin{aligned}
& P E R_{1}=1-\left(1-y_{\infty, 1}\right)^{L_{p}} \\
& P E R_{2}=1-\left(1-y_{\infty, 2}\right)^{L_{p}}
\end{aligned}
$$

where $L_{p}$ is the packet size.

In our proposed UEP transmission scheme, our goal is to maximize the average PSNR by adjusting selection probability $p_{1}$ and the number of the first part's bit-plane L within the required coding overhead $\varepsilon_{\max }$.

$$
P S N R_{o p t}=\max _{\left\{k, p_{1}\right\}: \varepsilon<\varepsilon_{\max }}\left\{P S N R_{\text {avg }}\right\}
$$

This problem is a classical convex optimization problem. Here we adopt descent method to solve it [10]. The solution algorithm is summarized as follows.

1) I/O definition: The input parameters include the compressed image data \{bit-plane 1, bit-plane 2, ---, bit-plane $N$ \}, the degree distribution $\Omega$ of LT code. The output parameters are $p_{1}$ and $k$.

2) Optimum Search: For $k$ from 1 to N, do the following steps.
Initialize $p_{c}$.

$\Delta=\operatorname{sign}\left(\operatorname{PSNR}_{\text {avg }}\left(p_{c}+\lambda\right)-\operatorname{PSNR}_{\text {avg }}\left(p_{c}\right)\right)$

$p_{n}=p_{c}+\Delta \lambda$;

while $\operatorname{PSNR}_{\text {avg }}\left(p_{n}\right)>\operatorname{PSNR}_{\text {avg }}\left(p_{c}\right)$

$p_{c}=p_{n}$

$p_{n}=p_{c}+\Delta \lambda$;

End while

Add element $\mathrm{p}_{\mathrm{c}}$ to the array $\mathrm{P}$.

End For

3) Output: find the maximal element in array $\left(\operatorname{PSNR}_{\text {avg }}(P)\right)$, record this index as $k, P(k)$ as $p_{1}$.

In our optimization algorithm, $\lambda$ is the step of descent method, function $\operatorname{PSNR}_{\text {avg }}(x)$ can be obtained according to Equation 1, Equation2, Equation 3, Equation 4, Equation 5 and Equation 6. The complexity of this algorithm is very low. The total iteration of our algorithm is

$$
\frac{(N-1)}{\lambda}
$$

Typically, the number of bit-planes of the compressed image $N$ is less than 30 . If we choose the precision of the step $\lambda$ as 0.01 , the total iteration of our algorithm will be 3000 , which is a practical level for most CPUs.

\section{Simulation}

In this section, the simulation is conducted to verify our proposed UEP image transmission scheme.

The first experiment is conducted to reveal that the average image quality is related to two parameters in our scheme: the number of bit-planes in the first part $\mathrm{L}$, and the selection probability of the first part compressed image $p_{1}$.

In this experiment, the packet size, the coding overhead and the total packet number are set as 2048 bits, 1.9 and 30 respectively. The lenna512×512 image is selected. The degree distribution in this experiment is chosen as follows [4].

$$
\begin{aligned}
\Omega(x)= & 0.007969 x+0.493570 x^{2} \\
& +0.166220 x^{3}+0.072646 x^{4} \\
& +0.082558 x^{5}+0.056058 x^{8}+0.037229 x^{9} \\
& +0.055590 x^{19}+0.025023 x^{64}+0.00313 x^{66} .
\end{aligned}
$$

To be easier illustration, we define $k_{m}=p_{1} k$, where $\mathrm{k}$ is the number of the compressed image bits. As we can see in Fig. 6 , given the number of bit-planes in the first part $\mathrm{L}$, the average PSNR is the convex function of $\mathrm{Km}$. For every given $\mathrm{L}$, the optimal solution can be found at the only pole on the curve. This reveals that the condition of using our descent method is satisfied.

In the second experiment, our proposed UEP image transmission scheme is compared with the traditional EEP scheme. The parameters' setting is the same as the first experiment.

As we can see in Fig. 7, our proposed UEP image transmission scheme well outperforms the traditional EEP transmission, especially when the coding overhead is small. For example, our UEP scheme outperforms the EEP scheme 
$27 \mathrm{~dB}$ at the overhead of 1.2. Also our proposed UEP optimization algorithm avoids the complex numerical exhausted searching [11], resulting in a small computational overhead. Thus it is very applicable for the satellite communication.

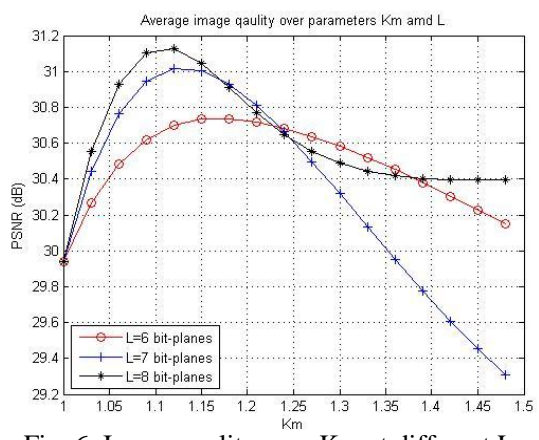

Fig. 6. Image quality over $\mathrm{Km}$ at different $\mathrm{L}$.

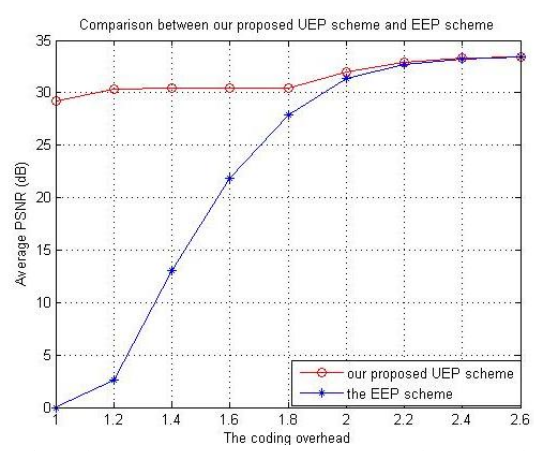

Fig. 7. Comparison between our proposed UEP scheme and EEP scheme.

\section{CONCLUSION}

This paper proposed a low-complexity UEP image transmission scheme over satellite. Different from the existing research work, our UEP image transmission scheme address the computational complexity of transmission scheme. First, a low-complexity image codec ISIPHT is selected as our image compression algorithm, which provides the almost same compression quality as JPEG2000 but much lower computational overhead and memory requirement. Second, the UEP optimization problem is simplified into the convex problem of a two parameters. Furthermore, the descent method instead of exhausting searching method is adopted to speed up the optimization process. With all these effort, our proposed UEP image transmission scheme is very applicable for image transmission applications over satellite. Finally, the simulation results are also presented to verify our advantage of UEP image transmission scheme over EEP scheme.

\section{REFERENCES}

[1] J. Byers, M. Luby, and M. Mitzenmacher, "A digital fountain approach to asynchronous reliable multicast," IEEE Jounal on Selected Areas in Communications, Oct. 2002.

[2] M. Luby, "LT codes," in Proc. 43rd IEEE Symp. Foundations Computer Science (FOCS), Nov. 2002, pp. 271-282.

[3] A. Shokrollahi, "Raptor codes," IEEE Trans. Inform. Theory, vol. 52, no.6, pp. 2551-2567, June 2006.

[4] N. Rahnavard, B. N. Vellambi, and F. Fekri, "Rateless codes with unequal error protection property," IEEE Trans. Inf. Theory, vol. 53, no. 53, pp. 1521-1532, Apr. 2007.

[5] N. Rahnavard and F. Fekri, "Finite-length unequal error protection rateless codes: Design and analysis," in Proc. GLOBECOM:St. Louis, MO 2005.
[6] D. Vukobratovic, V. Stankovic, D. Sejdinovic, L. Stankovic, and Z. Xiong, "Expanding windows fountain codes for scalable video multicast," in Proc. IEEE proceesing ICME 2008, pp. 77-80, June 2008.

[7] T. Ma, P. Shrestha, M. Hempel, D. Peng, and H. Sharif, "Low-complexity image coder/eecoder with an approaching-entropy quad-tree search code for embedded computing platforms," in Proc. International Conference on Image Processing (ICIP), Brussels, Belgium, 2011.

[8] T. Ma, M. Hempel, and H. Sharif, "A survey of energy-efficient compression and communication techniques for multimedia in resource constrained systems," IEEE Communications Surveys and Tutorials, 2012.

[9] W. C. Li, L. M. Ang, and P. S. Kah, "Survey of image compression algorithms in wireless sensor networks," in Proc. International Symposium on Information Technology, vol. 4, pp. 1-9, 26-28 Aug. 2008.

[10] S. Boyd and L. Vandenberghe, Convex Optimization, Cambridge, New York, Melbourne, Madrid, Cambridge University, 2004.

[11] D. Vukobratovic, V. Stankovic, D. Sejdinovic, L. Stankovic, and Z. Xiong, "Scalable video multicast using expanding window fountain codes," IEEE Transactions on Multimedia, vol. 11, no. 6, pp. 1094-1104, Oct. 2009.

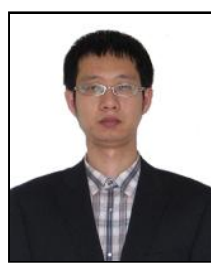

Tao Ma received the B.Sc. and M.Sc. degrees from Xian Jiaotong University, China in 2005 and 2008 respectively, both in electrical engineering. $\mathrm{He}$ received the Ph.D. degree in Department of Computer and Electronics Engineering, University of Nebraska-Lincoln, USA. He is currently a lecturer in state key laboratory on Integrated Services Networks (ISN), Xidian University, China. His research areas are Cross-layer design for QoS provisioning in wireless data networks, and multimedia distribution, ultralow power sensor network, and $4 \mathrm{G}$ network modeling and performance evaluation.

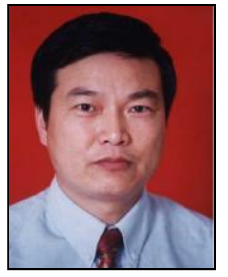

Zhanqi Xu received the $\mathrm{PhD}$ degree in communication and electronic system from Xidian University, China, in 1997. He currently serves as a professor at Xidian University, China. Dr. Xu had a one-year postdoctoral study at Hong Kong University of Science and Technology during the turn of this century. His interested areas include optical network, communication network modeling and performance evaluation.

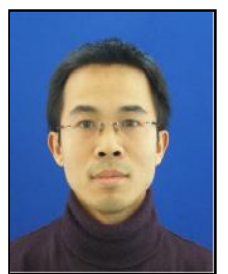

Xiaoyong Wei is the Dean of the department of Satellite Communication in $54^{\text {th }}$ Institute of Electronic and Technology Group of China. His research interest mainly focuses on the architecture design and development of the satellite communicaiton system. $\mathrm{He}$ was a receipent of the second award of the technology development of the $54^{\text {th }}$ Institute of Electronic and Technology Group of China.

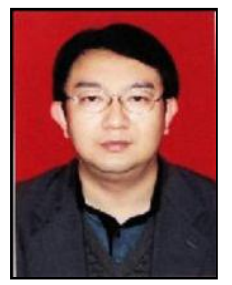

Fan Yang received BS and MS and Ph.D. degree in Xidian University in 1995 and 1998 and 2002 respectively. He is currently an associate professor in the Communication Engineering College of Xidian University. His research interests include high speed switching, optical switching, optical network and green communication network.

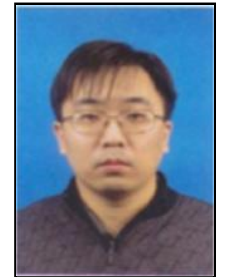

Zhe Ding received the B.E. and M.E. degrees in electrical engineering in the area of communications from the Xidian University, Xi'an, China in 1997 and 2005 , respectively. He joined the Xidian University in 1997. He is currently an Engineer in the State Key Lab of Integrated Service Networks in Xidian University. His research interests include the routing of the multi-layer or multi-domain network, the optimization the optical network 